\title{
Modélisation de la cavitation tridimensionnelle dans les pompes
}

\author{
J.L. Kueny, T. Maitre, J.L. Reboud \\ CREMHyG \\ Centre de Recherche et d'Essais de Machines Hydrauliques de Grenoble \\ INPG-ENSHMG \\ Domaine Universitaire \\ BP 95, 38402 St-Martin-d'Hères
}

\begin{abstract}
Nomenclature
$\psi \quad$ : fonction de courant
$V_{c} \quad$ : vitesse à l'interface de la poche
$P_{c_{5}} \quad$ : pression de vapeur

$V_{0}$ : vitesse de référence à l'amont du profil

$P_{0}$ : pression amont

$l_{c} \quad$ : longueur de la cavité

$\sigma_{c}=\left(P_{0}-P_{v}\right) /\left(0,5 \rho V_{0}^{2}\right):$ nombre de cavitation
\end{abstract}

\section{Introduction}

Pour la plupart des pompes, la cavitation est le phénomène critique qui limite la capacité d'aspiration. Elle impose la vitesse de rotation limite et le niveau d'implantation de la machine, ce qui entraîne souvent des contraintes de génie civil, d'encombrement ou de poids. Elle est aussi responsable d'instabilité de fonctionnement, de bruit et éventuellement d'érosion.

Si le tracé des roues et le circuit d'aspiration sont conçus correctement, les figures de cavitation qui sont à l'origine des chutes de caractéristiques de pompes au voisinage du débit nominal, sont le plus souvent les poches de vapeur qui se développent sur l'extrados des aubages. Le développement d'outils de prévision de ces poches, avec leur validation, est donc un objectif prioritaire pour les concepteurs et de nombreuses méthodes ont vu le jour, en particulier au CREMHyG depuis 1987.

Issues de techniques de prévision de poches de cavitation partielle bidimensionnelle sur des profils, ces méthodes ont été étendues au cas des poches $3 \mathrm{D}$ qui se développent sur les aubages de roues de pompe, dans un premier temps à partir d'une formulation quasi-3D du type S1/S2. Pour calculer des écoulements cavitants dans des pompes où les effets $3 \mathrm{D}$ sont mal représentés par les hypothèses du couplage méridien/aube à aube, la méthode a été introduite dans un code EULER 3D.

Un critère de prévision de la pression d'aspiration qui correspond aux chutes de caractéristiques de la pompe a été mis au point et validé sur quatre pompes de géométrie différentes.

\section{D modelling of cavitating flows in pumps}

The computation of $3 D$ attached cavities on pump blades using a numerical iterative technique is presented. The cavity shape is obtained by imposing a fixed pressure value on the corresponding stream-surface, as boundary condition in a $3 D$ flow calculation in the pump (SI/S2 or $3 D$ EULER code). A criteria for the prediction of the characteristic drop due to cavitaton is proposed. Results obtained for several pump geometries are discussed (SHF-pump, ns 20 and 80 pumps and the LH2 inducer of the SEP VULCAIN motor). 
Modèles 2D et validation

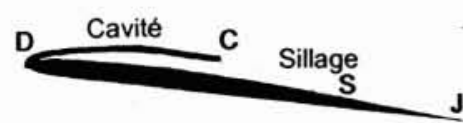

J

1. Modélisation $2 D$.

Les techniques de modélisation $3 \mathrm{D}$ des poches de cavitation sont, à l'origine, issues des modèles bidimensionnels qui ont été longuement utilisés pour des géométries du type venturi ou profil dans un tunnel de cavitation, voir par exemple [1]. Elles sont basées sur un calcul fluide parfait de l'écoulement extérieur éventuellement couplé à une modélisation des pertes visqueuses par le biais d'un coefficient de frottement ou d'une épaisseur de déplacement estimée avec un calcul de couche limite. L'épaisseur locale $b(x, y)$, dans la $3^{\mathrm{e}}$ direction peut être prise en compte par le calcul. Cette variation de l'épaisseur transversale, utilisée pour l'extension au calcul quasi-3D, voir ci-dessous, est déjà utile pour la validation du modèle $2 \mathrm{D}$ par comparaison à des expériences en tunnel de cavitation, pour la prise en compte des couches limites sur les parois latérales, lorsque les effets de confinement sont sensibles. Dans ces conditions, une fonction de courant $\psi$ est définie par

$$
\frac{\partial \psi}{\partial x}=-b \cdot V_{y} \quad \text { et } \quad \frac{\partial \psi}{\partial y}=b \cdot V_{x}
$$

$\psi$ est constant le long des frontières imperméables. A l'amont et à l'aval de la veine, l'écoulement est uniforme. En imposant la valeur $\psi_{p}$ de la fonction de courant sur le profil le calcul de l'écoulement est réalisé par une technique du type éléments finis, singularités ou volumes finis.

La condition de Kutta-Joukovski permet d'estimer la valeur $\psi_{p}$ de $\psi$ sur un profil placé dans l'écoulement. Ici, nous avons choisi d'imposer la direction de l'écoulement au bord de fuite $J$ du profil. Avec l'hypothèse de fluide parfait, l'équation pour la fonction de courant $\psi$ est linéaire. A partir de 2 solutions indépendantes du problème, la solution qui satisfait la condition de Joukowski est obtenue en recherchant la combinaison linéaire pour laquelle l'écoulement au bord de fuite $J$ du profil suit une direction imposée.

La géométrie de la poche est supposée stationnaire. La pression de vapeur dans la poche étant constante pour la plupart des fluides, la forme de la frontière de la cavité est obtenue en recherchant la ligne de courant $\psi=\psi_{p}$ qui est simultanément une isobare. Cette condition de pression constante se traduit en écrivant que la vitesse tangentielle

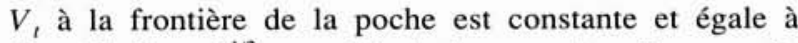
$V_{c}=V_{0}(1+\sigma)^{1 / 2}$; on obtient alors une deuxième condition limite pour $\psi$ :

$$
\partial \psi / \partial t=b V_{c} .
$$

La position du point $\mathrm{D}$ de détachement de la cavité est soit estimée par un critère du type décollement laminaire ou $C_{p}$ min dans le cas d'un bord d'attaque à forme arrondie, soit imposé au droit d'un décrochement de géométrie au bord d'attaque. On impose en $D$ deux conditions :

$$
\psi=\psi_{p} \quad \text { et } \quad V_{t}=V_{c} .
$$

L'arrière de la poche est modélisée par une portion de ligne de courant $C S$ raccordant de manière lisse le dernier point de la poche au profil ; ce «sillage numérique » peut être assimilé à une épaisseur de déplacement moyenne (moyenne spatiale et temporelle) de cette zone instationnaire, diphasique, turbulente et tridimensionnelle. La longueur du sillage est un paramètre empirique ajusté à partir de résultats expérimentaux dans des expériences caractéristiques du même type. Lorsque la pression de poche varie, cette longueur évolue soit proportionnellement à la longueur ou à l'épaisseur de la cavité soit conserve une longueur constante.

En imposant le point de détachement de la poche avec les longueurs de la poche et du sillage, le problème de la détermination de la position de l'interface et de la pression de poche (ou $\sigma$ ) est non linéaire et peut être résolu par la procédure itérative qui suit. A partir d'une position initiale de la ligne poche-sillage, en imposant une pression constante sur cette ligne, on estime la vitesse de transpiration sur cette frontière avec la pression de poche, en calculant l'écoulement potentiel extérieur. La frontière de la cavité est alors déplacée sur la ligne de courant qui part du point de décollement imposé de la poche. La convergence est obtenue en 4 à 5 itérations.

La validation expérimentale de ces techniques de modélisation de poche de cavitation est nécessaire en particulier pour confirmer les choix empiriques introduits au niveau de la longueur du "sillage numérique " et éventuellement pour le point de détachement de la poche. Ces expériences sont réalisées dans des tunnels de cavitation avec des profils ou des géométries du type venturi. Des mesures de pression en paroi sous les poches et dans la zone de sillage avec des mesures de vitesses par vélocimétrie laser dans l'écoulement sont utilisées pour ces comparaisons. Récemment [2], une nouvelle technique a été mise au point au CREMHyG, associant un éclairage par tranche laser et une technique d'analyse d'image, et permet maintenant d'accéder à la forme moyenne de la poche (voir figures 2 et 3 ).

Des résultats typiques de telles comparaisons sont représentés sur les figures 4 et 5 . Les formes de poches sont bien prévues par le calcul dans la zone modélisée à pression constante. Compte tenu des fortes fluctuations dans la zone arrière des poches, voir l'écart type de l'épaisseur de poche sur la figure 3, le « sillage numérique » est une ligne fictive qui n'a pas à être comparée à la position moyenne de la cavité dans cette zone, mais à cette position augmentée d'une épaisseur de déplacement liée aux fluctuations. 


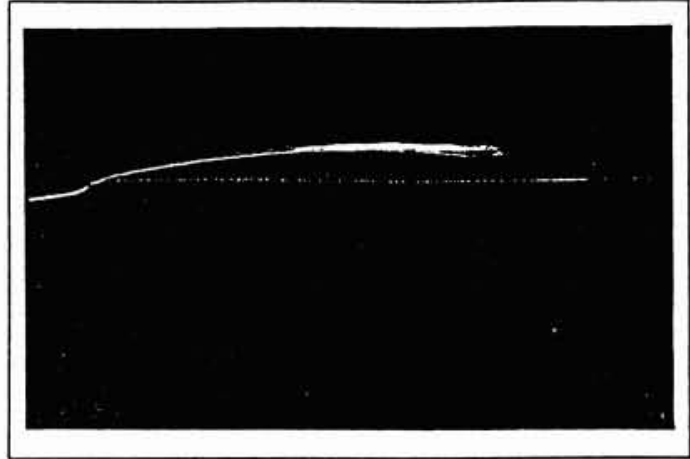

2. Superposition des images vidéo obtenues avec et sans cavité avec l'interface calculée par une procédure d'extraction de contour.

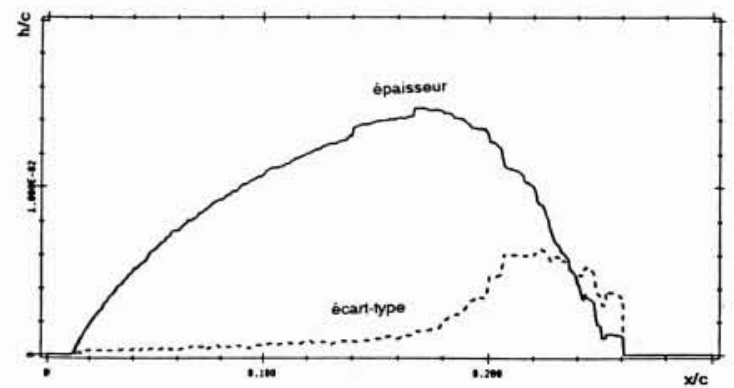

3. Epaisseur moyenne de la poche de cavitation avec son écar type.

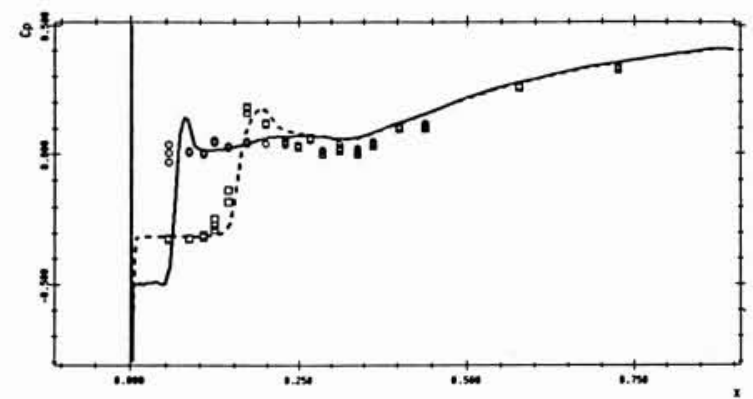

4. Comparaison calculs/expériences. Evolution de la pression sur un profil pour deux valeurs du nombre de cavitation.

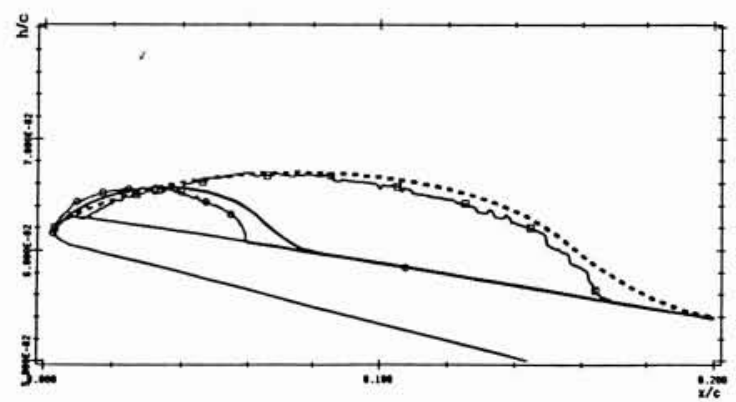

5. Formes des poches calculées et mesurées pour l' exemple de la figure 4.

\section{Modèle 3D - Code S1/S2}

Dans une première approche tridimensionnelle de la poche de cavitation se développant sur un extrados de pompe, la technique précédente a été directement transposée dans un calcul S1/S2 [3], [4]. L'étape du calcul méridien fournit la position, supposée axisimétrique des tubes de courant. Le modèle bidimensionnel est utilisé dans chaque section $\mathrm{S} 1$ aube-à-aube et permet d'estimer pour chaque tube de courant une loi longueur de poche en fonction du sigma de cavitation. Dans les cas sans effet thermodynamique, cette loi est une bijection et la longueur de poche qui correspond au sigma de la pompe est alors aisément obtenue par interpolation sur ces courbes dans chaque tube de courant (voir figure 6). Les déformations de la poche étant en général relativement faibles, une correction du calcul méridien n'est en général pas utile.

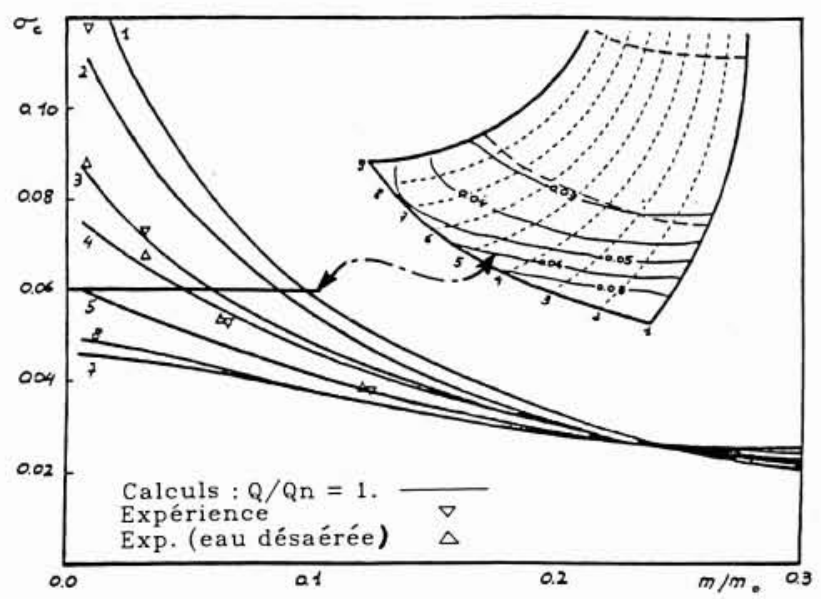

6. Technique d'évaluation des formes de poche de cavitation à partir des différentes courbes $I_{c}(0)$, calculées par le code SI/S2, pour la pompe SHF.

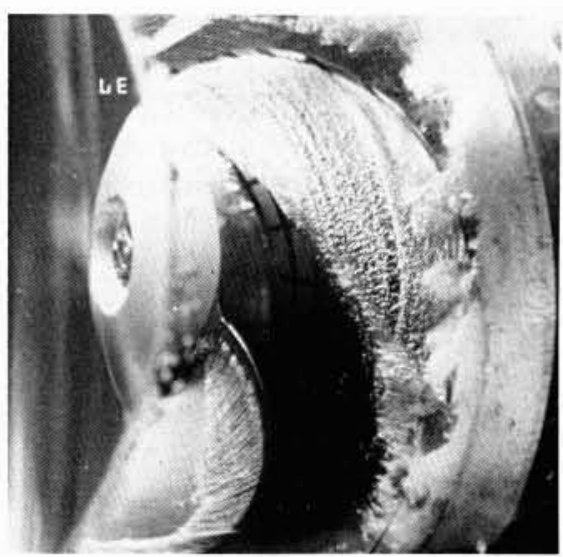

7. Poche de cavitation se développant sur I' inducteur SEP LH2 du moteur Vulcain. $Q / Q n=1$. Un quadrillage tracé sur l'aube permet d'évaluer l'étendue de la poche. 
La validation expérimentale est possible lorsqu'on dispose de visualisations exploitables quantitativement des poches qui se développent sur les aubages tournants. C'est le cas, par exemple de l'inducteur LH2 du moteur Vulcain de SEP pour lequel des visualisations complètes de ces poches avec l'évolution de leur forme en fonction du NPSP ont été réalisées au CREMHyG pour différents débits (fig. 7, p. précéd.).

\section{Modèle 3D-EULER}

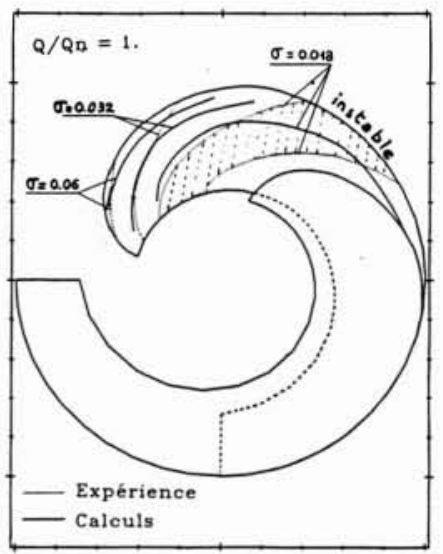

8. Comparaison calculs/expériences avec l'inducteur $\mathrm{LH} 2$ en fonction de la pression d'aspiration. $Q^{\prime} Q n=1$.

Sur la figure 8 , on peut voir que le modèle de prévision de poche, construit avec le code $\mathrm{S} 1 / \mathrm{S} 2$, fournit des résultats satisfaisants pour environ $2 / 3$ de la poche, du côté moyeu de l'aubage. Pour la partie de la poche en bout de pale, un écart systématique est observé. Un décalage d'incidence dû à un effet 3D lié à la forme en flèche de l'aube peut expliquer l'écart sur les petites poches. Cet effet ne peut pas être pris en compte par le calcul S1/S2. Pour les grandes poches, l'écoulement de jeu qui élève la pression au voisinage du carter, amplifie cet écart en bout de pale.

La modélisation des poches de cavitation, directement dans un code de calcul d'écoulement tridimensionnel, est actuellement réalisée et devrait permettre d'améliorer les prévisions pour des écoulements du type ci-dessus. Un code EULER 3D [6], basé sur une formulation de CLEBSCH, développé par EDF est utilisé pour estimer l'écoulement extérieur. La forme de la cavité est estimée itérativement. Dans l'étape de calcul de l'écoulement 3D extérieur, cette frontière de cavité est imposée. Pour modifier la géométrie de l'interface, un module de calcul de poche sur les surfaces axisymétriques du maillage (fig. 9), qui ne sont plus cette fois-ci des tubes de courant a été développé. L'effet tridimensionnel, lié à la composante transversale de la vitesse, estimée par le calcul EULER 3D extérieur, est pris en compte dans la formula-

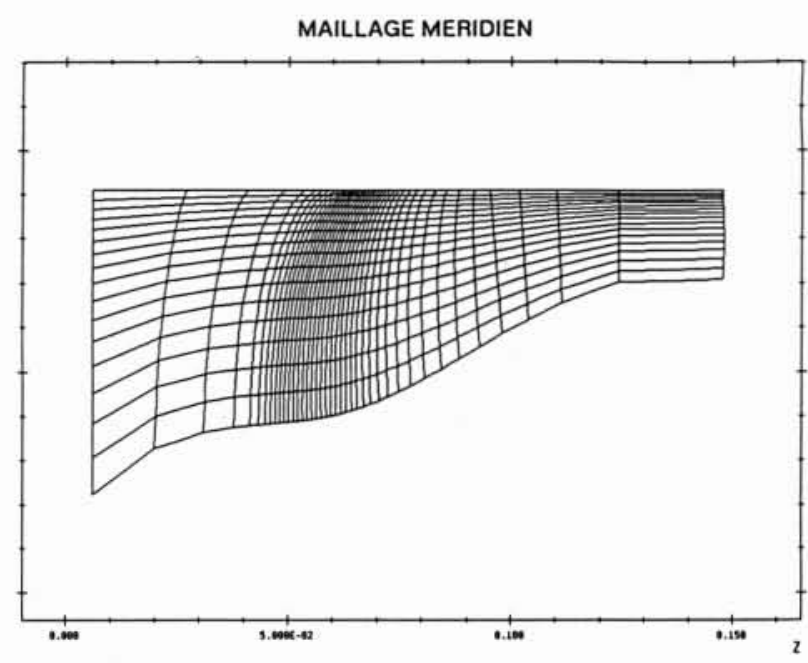

9. Maillage utilisé pour le calcul EULER $3 D$ de l'inducteur SEP LH2 du moteur Vulcain.

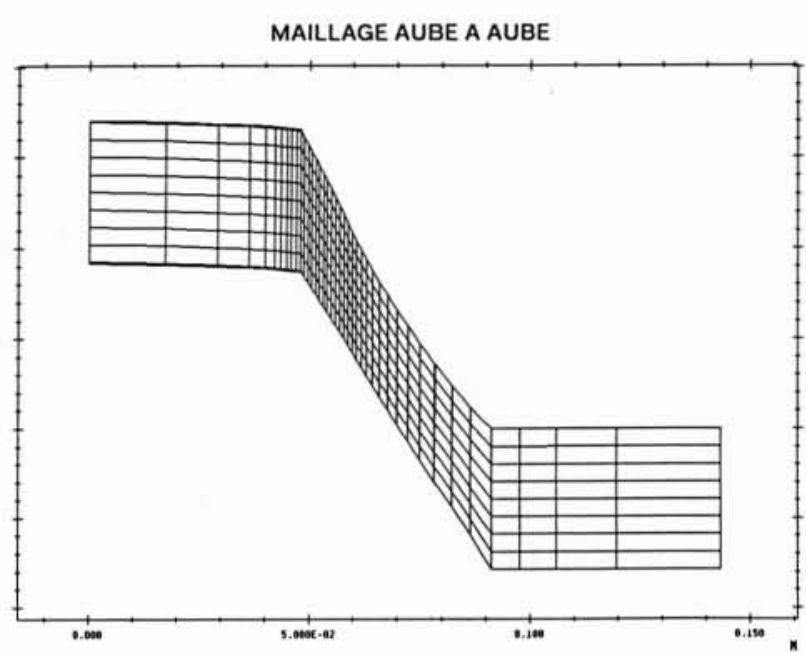

Surface axisymétrique du maillage, utilisée pour le calcul de la forme de poche de cavitation.

tion. La forme de la poche sur chaque surface axisymétrique est calculée par des itérations du même type que dans le cas bidimensionnel. La procédure itérative a été adaptée pour obtenir directement la longueur de poche correspondant à une pression d'aspiration imposée et ainsi éviter l'interpolation sur les courbes $l_{c}(\sigma)$ qui auraient dû être estimées pour chaque surface.

La validation de cette nouvelle méthode est actuellement en cours avec la géométrie de l'inducteur LH2 de Vulcain. Un résultat de calcul à la fin de la première itération est présenté sur la figure 10. Déjà, pour ce calcul non convergé, on constate une amélioration du comportement de la poche vers l'extrémité de l'aubage. Cette évolution est directement liée au comportement tridimensionnel du champ des vitesses au voisinage du bord d'attaque, côté carter, voir figure 11, qui ne peut typiquement pas être représenté par un calcul S1/S2.

Ce résultat doit être confirmé par le calcul complet en cours actuellement. 


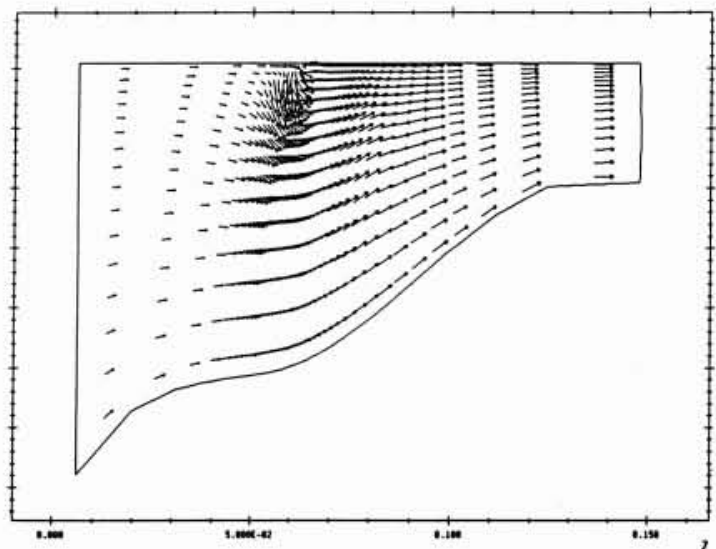

10. Projection sur la vue méridienne du champ des vitesses calculé par le code EULER 3D. Extrados de l'aubageInducteur SEP LH2 du moteur VULCAIN.

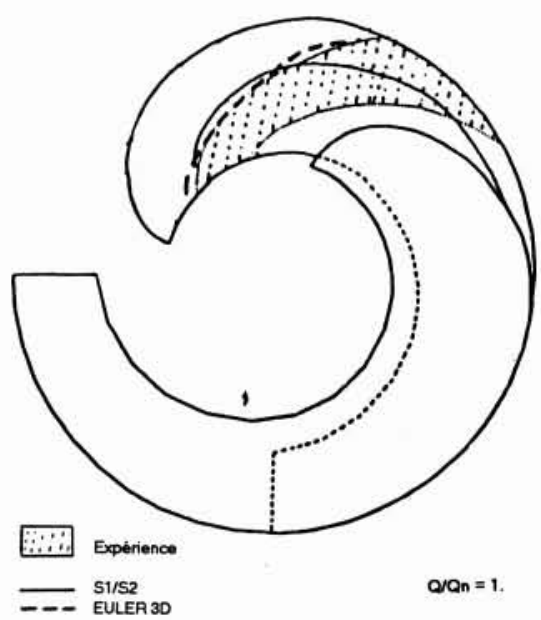

11. Comparaison calcul S1/S2, calcul EULER $3 D$ (non convergé) et expérience, pour $o=0,018$. Inducteur SEP LH2 du moteur VULCAIN.

\section{Critère de prévision des chutes de caractéristiques}

Un critère de prévision des chutes de caractéristiques, basé uniquement sur la forme de la poche de cavitation a été mis au point. En pratique, pour la plupart des configurations de pompe, on constate que le blocage est obtenu lorsque la longueur de l'arrière de la cavité atteint le col, au droit du recouvrement de la pale précédente. Une faible diminution de sigma entraîne alors une grande extension de la poche et celle-ci est aspirée dans le canal inter-aube et ressort au bord de fuite. La cavitation affecte alors le champ de vitesse à la sortie et on observe la chute des caractéristiques de la pompe, suite à la dégradation du triangle de vitesse de sortie. Quand la cavité se développe dans la zone avant recouvrement des aubes, les pertes de la machine ne sont pratiquement pas affectées et le calcul $\mathrm{S} 1 / \mathrm{S} 2$, basé sur une hypothèse fluide parfait, représente correctement l'écoulement au voisinage du débit nominal. Le sigma correspondant au passage du col par une proportion significative de la poche de cavitation permet donc d'estimer le NPSH critique correspondant aux chutes de caractéristiques.

Suivant la géométrie de la pompe, les poches peuvent avoir un aspect tridimensionnel plus ou moins important. A même pression amont, la longueur de la cavité peut être pratiquement constante le long de l'envergure de l'aube, voir figure 6 ou 12 ou varier fortement du moyeu à la ceinture, voir figure 14. Le tracé du développement des poches sur l'ensemble de l'envergure est nécessaire pour utiliser correctement ce critère géométrique de chute de performances, en effet, il doit correspondre au passage dans le col d'une portion de poche de taille suffisante pour entraîner le blocage. Les résultats obtenus avec la pompe SHF, deux pompes de ns 20 et 80 et d'un inducteur de la pompe LH2 du moteur VULCAIN de SEP sont présentés sur les figures 12 à 15 .

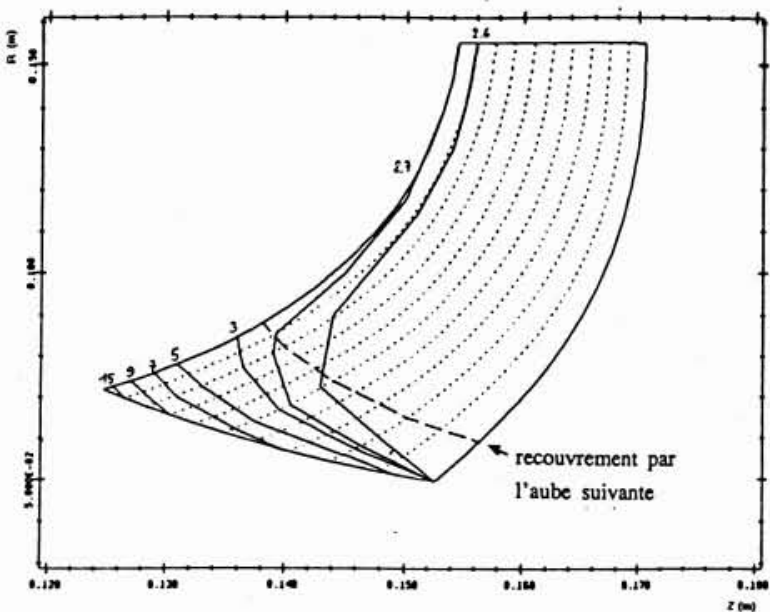

12. Géométrie des poches de cavitation. Pompe ns 20 , 10 aubes, Qn, $1475 \mathrm{tr} / \mathrm{mn}$. NPSH : 15, 9, 7, 5, 3, 2,7 et 2,6 mCE (taille de poche croissante).

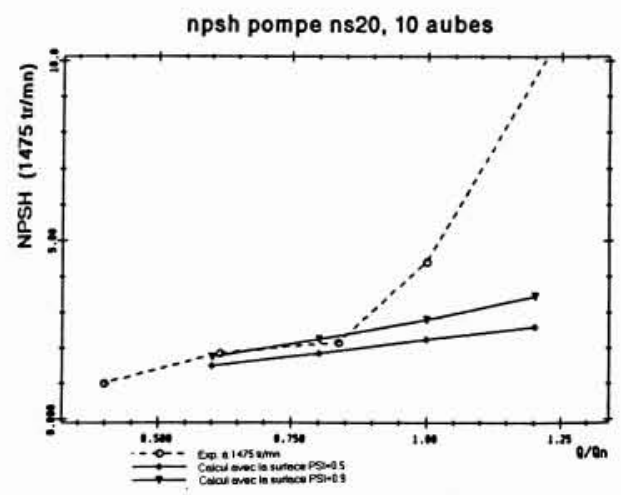

13. Prévision du NPSH critique correspondant aux chutes de caractéristiques. Pompe ns 20. 


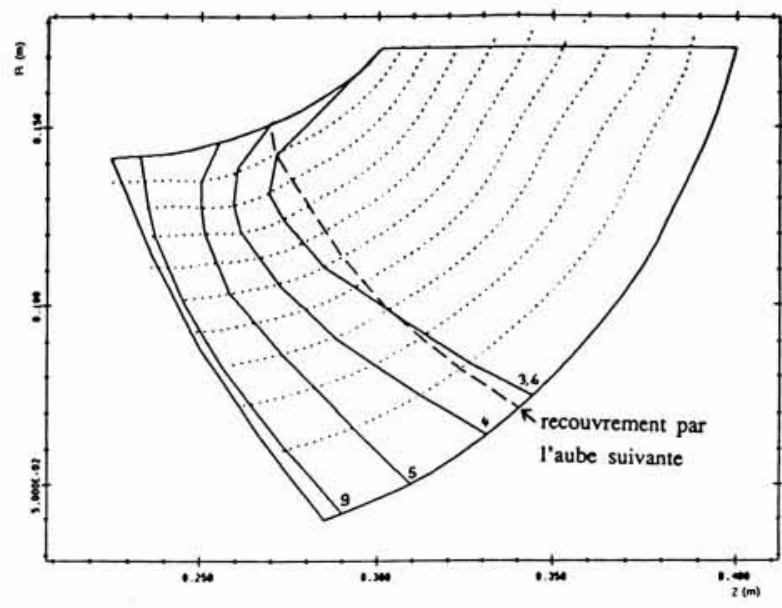

14. Géométrie des poches de cavitation. Pompe $\mathrm{ns} 80$, Qn, $1150 \mathrm{tr} / \mathrm{mn}$. NPSH : 9, 5, 4 et 3,6 mCE (taille de poche croissante).

\section{Conclusion et perspectives}

Les modèles tridimensionnels de prévision de poche de cavitation, présentés ci-dessus, sont construits à partir d'une hypothèse de forme stationnaire constituée d'une première partie de poche à pression constante ou imposée qui se raccorde au profil par une zone arrière dite « sillage numérique ". La modélisation numérique de l'écoulement extérieur est basée sur une formulation « fluide parfait ", quasi tridimensionnelle $\mathrm{S} 1 / \mathrm{S} 2$, ou tridimensionnelle EULER. Ces modèles, avec le critère de prévision du NPSH critique correspondant à la chute des caractéristiques, ont été validés au voisinage du débit nominal des pompes.

Pour étendre les possibilités de cette technique, plusieurs voies nouvelles sont actuellement explorées au CREMHyG.

La prise en compte d'un effet thermodynamique, négligeable dans l'eau froide, mais important avec des fluides de pompes de moteur cryotechnique de fusée ou des hydrocarbures, est réalisée en modélisant simutanément l'écoulement liquide à l'extérieur de la poche, l'écoulement de vapeur à l'intérieur de la poche, les échanges de chaleur et de masse à l'interface liquide-

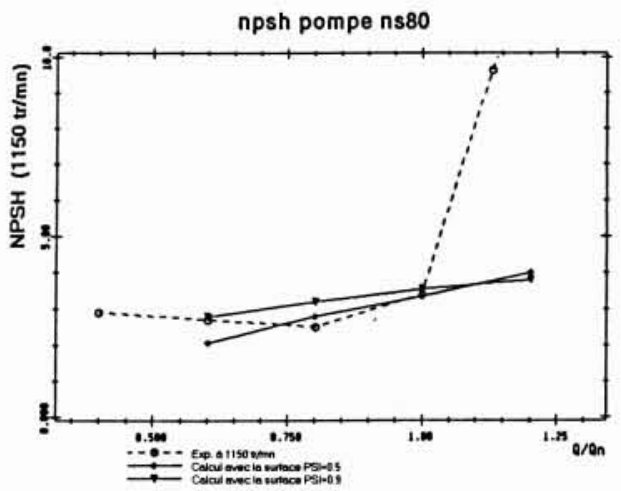

15. Prévision du NPSH critique correspondant aux chutes de caractéristiques. Pompe ns 80. vapeur et le transport de la chaleur dans le liquide et la vapeur [8]. La modélisation de la turbulence au voisinage de l'interface est nécessaire dans ce cas. Le modèle est mis au point dans une géométrie bidimensionelle et sera transposé aux codes tridimensionnels.

Pour élargir le domaine de validité de la méthode, la modélisation de poches de cavitation est développée dans un code d'écoulement 3D Navier Stokes, prenant en compte les effets visqueus et la turbulence (code COPERNIC développé avec SEP et ONERA). Dans ce cas, la modélisation de la poche est directement réalisée sous forme d'une condition imposée pour la pression à l'interface liquide-vapeur dans le calcul 3D d'écoulement; la géométrie de la poche est obtenue au cours des itérations par déformation de la frontière. On retrouve ici, comme ci-dessus, le problème d'interaction cavitation-turbulence.

Le développement d'un code de calcul d'écoulements diphasiques instationnaires permet d'aborder l'analyse et la prévision d'effets instationnaires de la cavitation (version 2D actuellement [9]). Avec ce type de modèle il sera possible d'envisager la prévision de configurations d'écoulements cavitants instationnaires, par exemple sillage de poche, cavitation par bulles, vortex cavitant ou pulsation de torche.

\section{Remerciements}

Nous remercions la Société Européenne de Propulsion (SEP) et le Centre National d'Etudes Spatiales (CNES), qui ont financé la plupart des études présentées ci-dessus.

\section{Références}

[1] Kueny J.L., Reboud J.L., Desclaux J. - "Analysis of Partial Cavitation : Image Processing and Numerical Prediction », ASME Cavitation Symposium, Portland 1991.

[2] Kueny J.L., Joussellin F., Boutet J.M. - « Analyse d'image et cavitation développée sur un profil ", Colloque national de visualisation et de traitement d'images, Lille 1990.

[3] Schultz J.L. - «Modélisation de la cavitation partielle dans les pompes ", Thèse de doctorat de l'INPG, 1988.

[4] Kueny J.L., Desclaux J. - « Etude théorique de la cavitation dans les pompes et inducteurs ", Revue Française de Mécanique n` 1989-1.

[5] Kueny J.L., Desclaux J. — « Theorical Analysis of Cavitation in Rocket Engine Inducers », ASME Pumping Machinery Symposium, San Diego 1989.

[6] El Ghazzani E.M. - "Calcul de l'écoulement dans une turbomachine à l'aide des variables de Clebsch et par une méthode d'éléments finis ", Thèse de doctorat de l'ECL, 1990.

[7] Mattre T., Kueny J.L., «Three-Dimensional Models of Cavitation in Rocket Engine Inducers ", ASME Fluid machinery Forum, Toronto 1990.

[8] Reboud J.L., Sauvage Boutar E., Desclaux J., « Partial Cavitation Model for Cryogenic Fluids ", ASME Cavitation and Multiphase Flow Forum, Toronto 1990.

[9] Delannoy Y., Kueny J.L. - « Two Phase Flow Approach in Unsteady Cavitation Modelling ", ASME Cavitation and Multiphase Flow Forum, Toronto 1990. 